\title{
Supplementary Material - 6
}

Tasks

Visual depictions of the tasks are presented here for visualisation processes. See (Foster et al.,2014) (DOI 10.3758/s13421-014-0461-7) for the a more exact representation of the tasks.

\section{OSPAN}

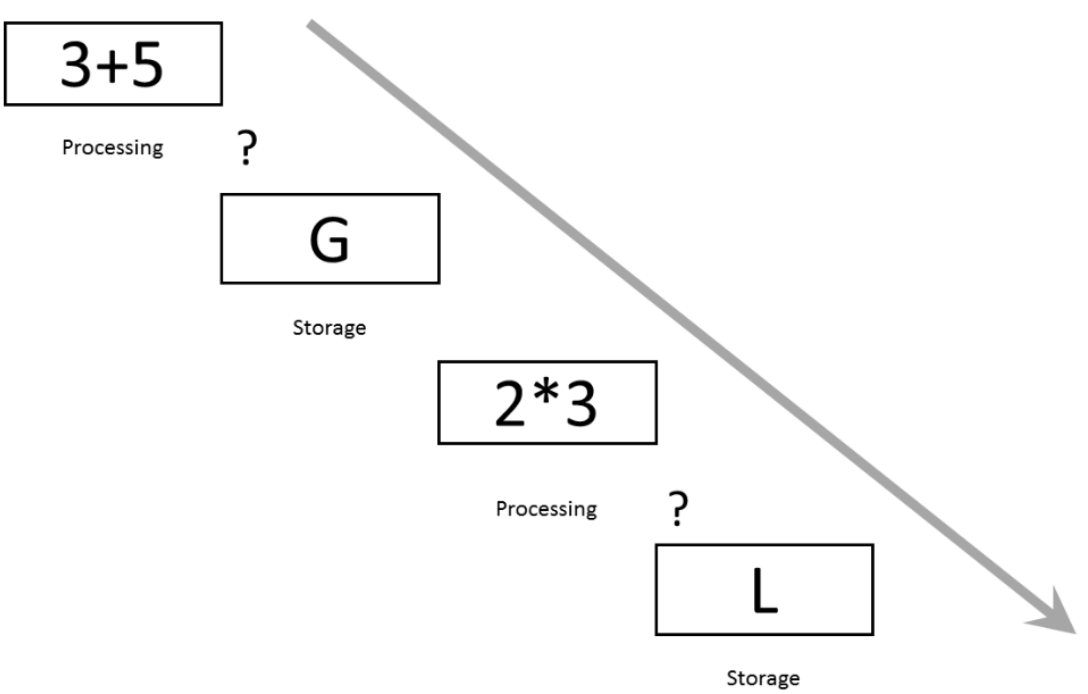

S6 Fig 1 - Visual depiction of the ospan task

SYMSPAN
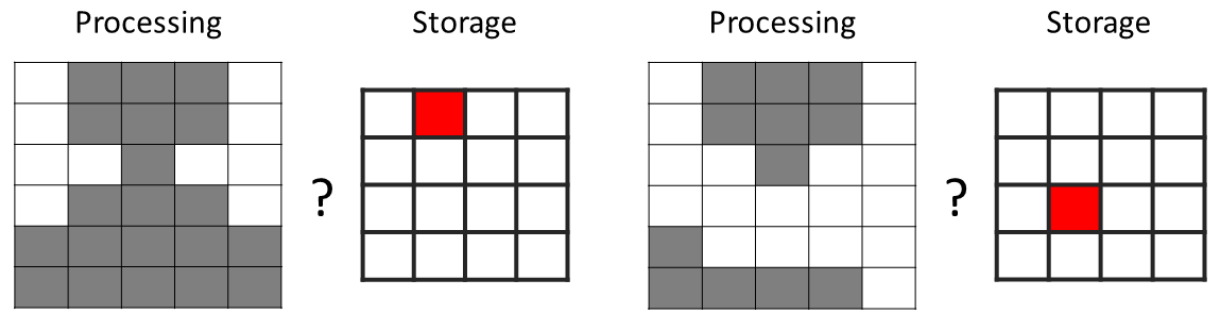

S6 Fig 2 - Visual depiction of the symspan task 


\section{ROTSPAN}

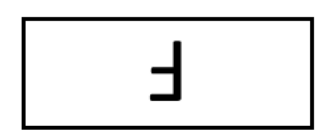

Processing ?

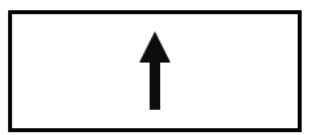

Storage

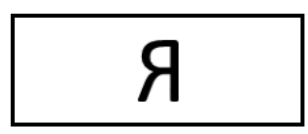

Processing

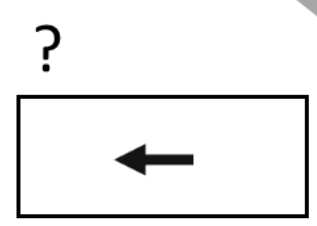

Storage

S6 Fig 3 - Visual depiction of the rotspan task 